\title{
Strong configuration mixing due to dielectric confinement in semiconductor nanorods
}

\author{
J. I. Climente, M. Royo, J. L. Movilla, and J. Planelles* \\ Departament de Química Física i Analítica, Universitat Jaume I, Box 224, E-12080, Castelló, Spain
}

(Received 30 December 2008; published 1 April 2009)

\begin{abstract}
The interaction between electron-electron and electron-hole pairs in semiconductor nanorods embedded in dielectric media is investigated using a configuration-interaction method. Contrary to spherical quantum dots of similar volume, the dielectric confinement is shown to bring nanorods into a regime of strong configuration mixing. The wave functions are particularly sensitive to such mixing, which leads to qualitative changes in the electronic and optical properties of the rods.
\end{abstract}

DOI: 10.1103/PhysRevB.79.161301

PACS number(s): 73.21.La, 77.22.Ej, 78.67.Hc, 71.27.+a

Semiconductor quantum rods or nanorods (NRs) are colloidal quantum dots with strong radial confinement and variable length. ${ }^{1,2}$ Even though NRs were first synthesized long after spherical nanocrystals (NCs), it was soon realized that their anisotropic shape posed many benefits for optical and transport applications. ${ }^{3}$ This prompted a large number of studies, often revealing characteristic physical phenomena which stem from the weak longitudinal confinement, for NRs bridge the gap between zero-dimensional and onedimensional quantum-confined systems. ${ }^{4-10}$

Similar to NCs, NRs are usually embedded in insulating media, whose dielectric constant is much lower than that of the semiconductor structure. This dielectric mismatch gives rise to a so-called dielectric confinement, which greatly enhances the Coulomb interactions inside the semiconductor. ${ }^{11,12}$ In spherical NCs, however, owing to the strong quantum confinement, Coulomb interactions are usually a first-order perturbation effect for the low-lying states. ${ }^{13}$ Furthermore, a strong spatial confinement leads to compensations between electron and hole charge distributions, ${ }^{14,15}$ so that the optical properties are barely affected by the dielectric environment.

None of these restrictions apply to NRs, where the longitudinal confinement may be fairly weak. Indeed, the dielectric confinement has been held responsible for the large variation in the optical gap of CdSe NRs as compared to the transport one., ${ }^{76}$ What is more, NRs are the zerodimensional counterpart of quantum wires, where variations in the dielectric confinement have been shown to induce drastic changes in the binding energy and oscillator strength of excitons, thus enabling Coulomb interaction engineering. ${ }^{17-19}$ One may then wonder to which extent the single-particle and perturbational treatments of Coulomb interactions that dominate the literature of NRs (Refs. 6-10) provide a valid description of the optoelectronic properties.

In this work, we perform a theoretical study of the effect of the dielectric confinement on interacting particles (two electrons or one electron and one hole) confined in a semiconductor NR. A numerical procedure is used which allows us to estimate the effect of the dielectric environment for arbitrary three-dimensional potentials, thus addressing realistic geometries. Electron correlations are then accounted for exactly using an effective mass-configuration-interaction (CI) scheme. We go beyond energetic effects and illustrate the influence of the dielectric confinement on the wave function, as well as on derived properties such as the electronhole recombination probability.
The fully three-dimensional model allows us to compare the behavior of rods with that of spherical dots. In the absence of dielectric mismatch, both systems may be well described by a perturbational model of the Coulomb interaction. However, with increasing mismatch, electronic correlations in the rod can reach very high values. This is made possible by the rod shape. The small radius makes the charge confined inside very sensitive to the external dielectric medium, while the weak longitudinal confinement renders the system prone to configuration mixing. It then follows that the physics of NRs in dielectric surroundings is far from the strong confinement picture. Signatures of the severe configuration mixing, such as enhanced exciton emission, the appearance of new optical modes, or Wigner localization of few-electron states, are predicted.

The electron and hole single-particle states are described with effective-mass Hamiltonians which, in cylindrical coordinates and atomic units, read as ${ }^{20}$

$$
\begin{aligned}
\mathcal{H}_{i}= & -\frac{1}{2}\left[\frac{1}{\rho} \frac{\partial}{\partial \rho}\left(\frac{\rho}{m_{\rho, i}^{*}(\rho, z)} \frac{\partial}{\partial \rho}\right)+\frac{\partial}{\partial z} \frac{1}{m_{z, i}^{*}(\rho, z)} \frac{\partial}{\partial z}\right. \\
& \left.-\frac{m_{i}^{2}}{\rho^{2} m_{\rho, i}^{*}(\rho, z)}\right]+\hat{V}_{i}(\rho, z),
\end{aligned}
$$

where $i=e, h$ is a subscript denoting electron or hole, respectively. $m_{i}$ is the azimuthal angular momentum and $\hat{V}_{i}(\rho, z)$ is the spatial confinement potential, which is zero inside the nanostructure and $V_{i}^{\text {out }}$ outside. $m_{\rho, i}^{*}(\rho, z)$ and $m_{z, i}^{*}(\rho, z)$ are the position-dependent effective masses in the plane and along the vertical direction. For electrons we use isotropic masses $m_{\rho, e}^{*}(\rho, z)=m_{z, e}^{*}(\rho, z)$. For holes, however, the mass anisotropy is important, as it is responsible for the heavy-hole to light-hole ground-state transition that occurs as the aspect ratio of the nanostructure increases. ${ }^{7,21}$ Thus, for heavy holes we use $m_{\rho, h}^{*}=1 /\left(\gamma_{1}+\gamma_{2}\right)$ and $m_{z, h}^{*}=1 /\left(\gamma_{1}-2 \gamma_{2}\right)$, while for light holes we use $m_{\rho, h}^{*}=1 /\left(\gamma_{1}-\gamma_{2}\right)$ and $m_{z, h}^{*}=1 /\left(\gamma_{1}+2 \gamma_{2}\right)$. Here $\gamma_{1}$ and $\gamma_{2}$ are the Luttinger parameters. ${ }^{21}$ Hamiltonian (1) is integrated numerically using a finite differences scheme.

In order to determine the electrostatic potential $V_{\text {Coulomb }}(\mathbf{r})$, in a medium with spatially inhomogeneous dielectric constant $\varepsilon(\mathbf{r})$, we rewrite the Poisson equation in terms of volumetric source charges plus induced polarization charges, 


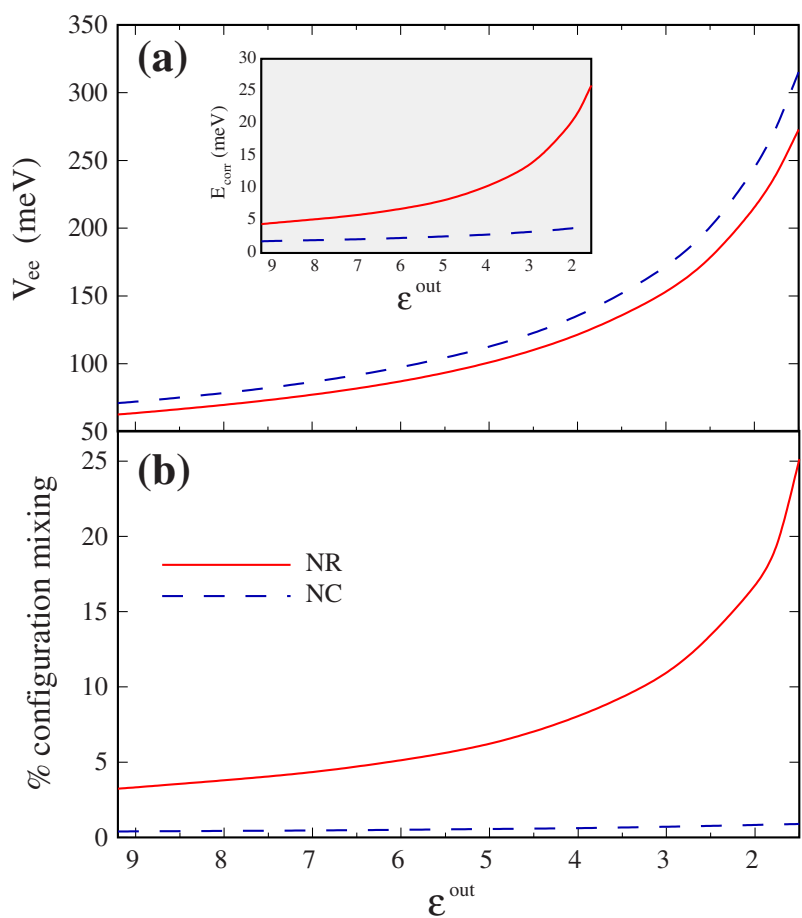

FIG. 1. (Color online) Two-electron ground-state properties in a NR (solid line) and a spherical NC (dashed line) of the same volume, as a function of the dielectric constant of the surrounding medium. (a) Electron-electron repulsion energy. (b) Weight of configuration mixing in the CI expansion. The inset represents the energy coming from Coulomb correlation (see text).

$$
\nabla^{2} V_{\text {Coulomb }}(\mathbf{r})=-4 \pi\left[\eta(\mathbf{r})+\eta_{p}(\mathbf{r})\right] .
$$

Here $\eta(\mathbf{r})$ is the source charge density, which is obtained from the electron eigenstates of Eq. (1), and $\eta_{p}(\mathbf{r})$ is the polarization charge density, which we calculate with a method equivalent to the induced charge computation one proposed by Boda et al. ${ }^{22}$ The Coulomb interaction is then taken into account by means of a CI procedure. The twoelectron (exciton) states are built as a linear combination of a large number of Slater determinants (Hartree products) obtained by filling in the single-particle eigenstates in all possible ways consistent with symmetry requirements, granting energy convergence within $0.1 \mathrm{meV} .{ }^{23}$ The fully interacting
Hamiltonian is diagonalized, exploiting orbital and spin symmetries. $^{24}$

In our calculations, we study a typical CdSe NR. The rod is composed by a cylinder of radius $R=2 \mathrm{~nm}$ and length $L$ $=8 \mathrm{~nm}$ attached to two hemispherical caps of radius $R$ $=2 \mathrm{~nm}$ in the extremes, giving a total length of $L_{\text {tot }}$ $=12 \mathrm{~nm}$ (see inset in Fig. 2). For comparison, we also study a spherical $\mathrm{NC}$ with roughly the same volume, i.e., $R$ $=3.15 \mathrm{~nm}$. The material parameters are $m_{e}^{*}(\rho, z)=0.13$ inside the structure and 1 outside and $\left(\gamma_{1}, \gamma_{2}\right)=(1.66,0.41)$ inside and $(1.0,0.0)$ outside. $^{25}$ The confinement potential of the outer media is set to $V_{e}^{\text {out }}=V_{h}^{\text {out }}=4 \mathrm{eV}$. The dielectric constant is fixed at $\varepsilon(\mathbf{r})=9.2$ inside and we vary the outer value $\varepsilon^{\text {out }}$ to simulate the effect of different surrounding media. For NRs, the ground state is assumed to be a light hole, ${ }^{7,21}$ so the $\mathrm{CI}$ is built on a subspace of light-hole states. Conversely, for spherical NCs we use heavy holes.

\section{ELECTRON-ELECTRON INTERACTION}

We start by investigating the two-electron case. Figure 1(a) compares the electron-electron repulsion energy $V_{\mathrm{ee}}$ for the ground state of the NR and the NC as a function of the dielectric mismatch. As can be seen, the repulsion energy in the NR experiences a similar enhancement to that of the spherical NC. For a usual surrounding medium of $\varepsilon^{\text {out }}=2.0$, $V_{\text {ee }}$ is over three times larger than the value one may expect neglecting the dielectric confinement. To quantify the amount of $V_{e e}$ which is not captured by simple perturbational descriptions of the interaction, in the figure inset we depict the correlation energy defined as $E_{\mathrm{corr}}=V_{\mathrm{ee}}^{0}-V_{\mathrm{ee}}$. Here $V_{\mathrm{ee}}^{0}$ is the expectation value of the Coulomb repulsion for the dominant configuration in the CI expansion. At this point, we observe a first difference between NRs and NCs. In the absence of dielectric mismatch, $E_{\text {corr }}$ is about twice larger for the NR. This is due to the weak confinement in the long axis direction. Furthermore, with increasing mismatch the ratio increases up to six, revealing much stronger correlation effects in NRs as compared to NCs.

Since the effect of correlations on the wave function is even more pronounced than that on the energies, in Fig. 1(b) we analyze the amount of configuration mixing experienced by the ground state, i.e., the weight of the CI expansion

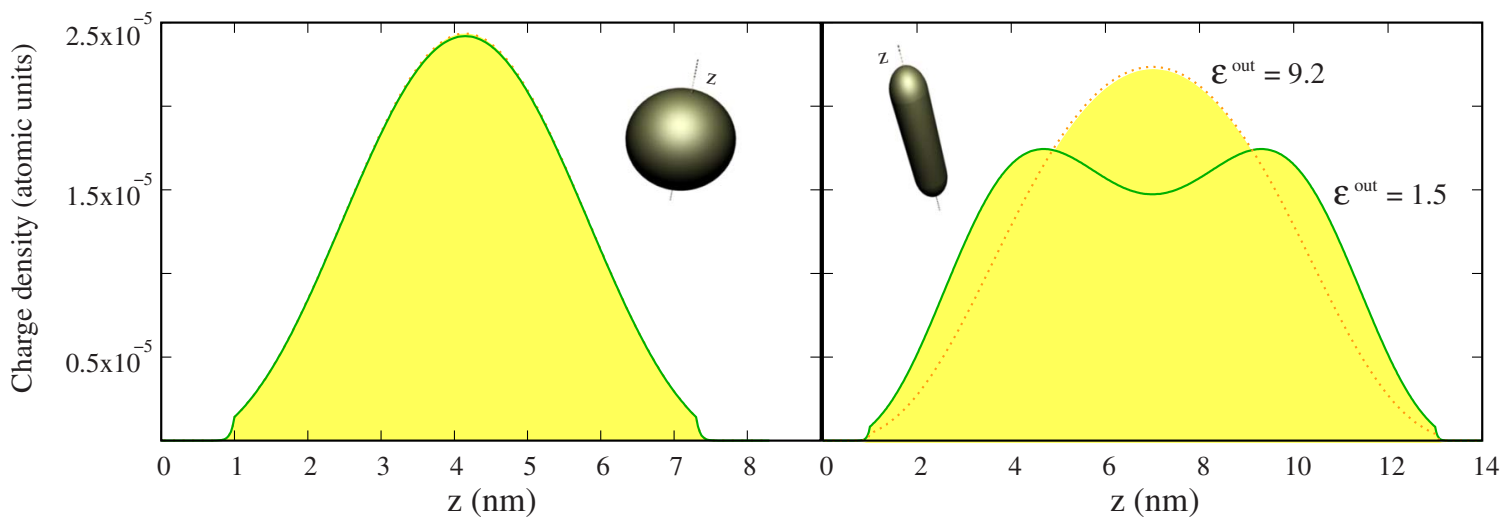

FIG. 2. (Color online) Two-electron ground-state charge density along the vertical axis of a NC (left) and a NR (right) with (solid line) and without (dotted line) dielectric confinement. The insets are schematics of the structures under study. 


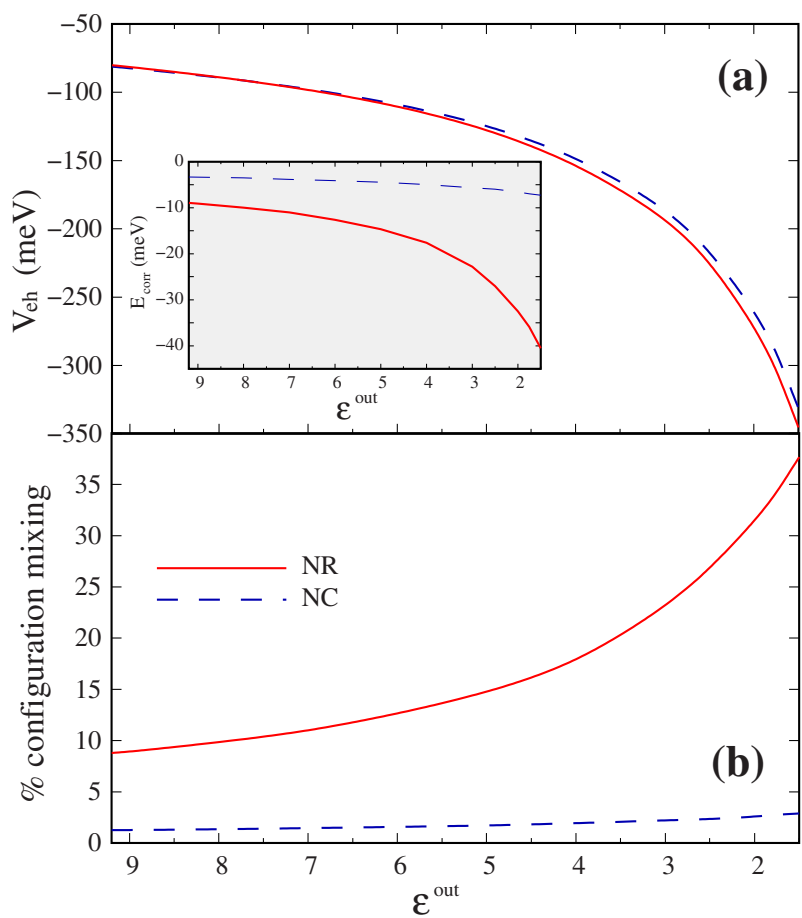

FIG. 3. (Color online) Same as Fig. 1, but for the exciton ground state.

which does not come from the dominant Slater determinant. The different behavior of NCs and NRs is now striking. The configuration mixing is minor for the spherical dot, ${ }^{13}$ but it becomes very large for dielectrically confined NRs.

The influence of the configuration mixing on the groundstate wave function is apparent in Fig. 2. In the figure, we represent the charge density of one electron along the vertical axis of the nanostructure after integrating over the coordinates of the second electron, for a spherical NC (left) and a NR (right). Solid and dotted lines are used for the cases with and without dielectric confinement. While the NC wave function remains essentially unaltered by the dielectric confinement, the NR wave function develops a valley in the center of the structure. This is a clear indication that Wigner localization is taking place in the NR, which will have direct implications for transport processes ${ }^{26}$ and shell-filling spectroscopy. ${ }^{27}$

\section{ELECTRON-HOLE INTERACTION}

Next we investigate the effect of the dielectric confinement on the exciton ground state. The results summarized in Fig. 3 are qualitatively the same as in the two-electron case, but correlations are now even stronger because of the large mass of the holes. Thus, in Fig. 3(a) one can see that the dielectric confinement enhances the electron-hole attraction (binding) energy $V_{\mathrm{eh}}$ at a similar rate for NRs and NCs. ${ }^{28}$ However the correlation energies are several times larger in the case of NRs (figure inset), for they undergo a sizable amplification with increasing dielectric mismatch. The effect of correlations is mostly felt through the wave function. As can be seen in Fig. 3(b), for a typical dielectric medium of

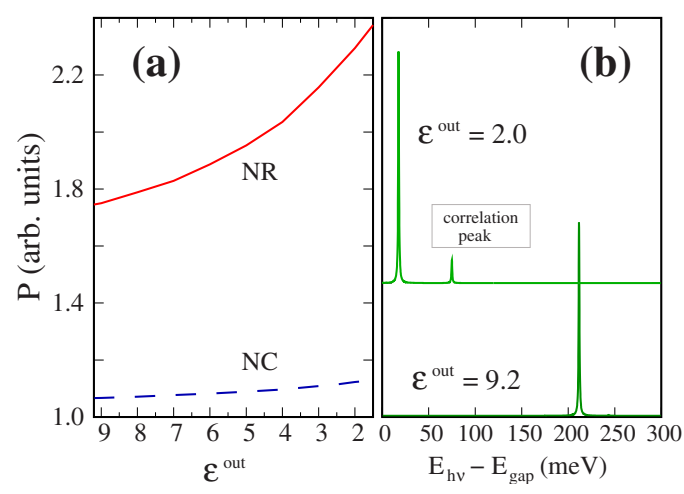

FIG. 4. (Color online) (a) Recombination probability of the ground-state exciton as a function of the dielectric constant of the surrounding medium, in a NR (solid line) and a NC (dashed line) of the same volume. (b) Absorption spectrum of the NR under a weak electric field, in the absence and presence of dielectric confinement. The spectra are offset for clarity. The high-energy peak at $\varepsilon^{\text {out }}$ $=2.0$ arises from Coulomb correlations

$\varepsilon^{\text {out }}=2.0$, the exciton ground state in the spherical NC is essentially given by the dominant Hartree product (an electron and a hole in the lowest single-particle orbitals), which validates the perturbation treatment. By contrast, the NR ground state contains over $30 \%$ of excited configurations admixed through Coulomb correlations. Obviously, this strong mixing modifies the exciton wave function. This has important effects on the optical properties of the NR which are not captured by usual perturbational descriptions. In what follows, we illustrate some of these effects.

In large size quantum dots, it has been shown that correlations enhance the exciton luminescence. ${ }^{29}$ We can show that this effect is also present in dielectrically confined NRs by calculating the electron-hole recombination probability, by means of the dipole approximation and a Fermi golden rule $^{30}$

$$
P_{\mathrm{gs}} \propto\left|\sum_{i} c_{i}^{\mathrm{gs}}\left\langle\phi_{i}^{e} \mid \phi_{i}^{h}\right\rangle\right|{ }^{2} \mathcal{P}^{2} .
$$

Here $c_{i}^{\text {gs }}$ is the ground-state CI expansion coefficient corresponding to the Hartree product formed by the electron and hole spin orbitals $\phi_{i}^{e}$ and $\phi_{i}^{h}$. $\mathcal{P}$ is the Kane matrix element. Figure 4(a) shows the exciton recombination probability in a $\mathrm{NR}$ and a NC as a function of the dielectric mismatch. As expected, the recombination probability in the NR is always larger than that in the NC. ${ }^{16}$ In addition, with increasing dielectric mismatch, the luminescence of the NR increases up to $40 \%$ : a clear signature of the strong correlations affecting the wave function.

Correlated wave functions are more sensitive to environmental perturbations than strongly confined ones. A manifestation of this is shown next. We simulate the absorption spectrum of a NR $\mathcal{A}(E)=\Sigma_{j} P_{j} \delta\left(E-E_{j}\right)$, where $E$ is the incident photon energy and $P_{j}$ is the recombination probability of the $j$ th exciton state, with energy $E_{j}$. The NR is subject to a moderate electric field of $50 \mathrm{kV} / \mathrm{cm}$ along the vertical direction, which breaks the parity symmetry, thus enabling optical transitions from the first-excited exciton state. Figures 4(b) 
compares the absorption spectrum without and with dielectric mismatch. In the absence of dielectric mismatch, when correlations are weaker, only the fundamental transition is visible. The first-excited transition is still negligible, as it involves the lowest single-electron orbital $\left(1 s_{e}\right)$, which is quasigerade, and the first-excited single-hole orbital $\left(2 s_{h}\right)$, which is quasiungerade. In the presence of dielectric mismatch a new transition appears. This transition corresponds to the first-excited exciton state because now the correlations lead to a strong mixing of the $1 s_{e} 2 s_{h}$ and $1 s_{e} 1 s_{h}$ configurations, the latter orbitals having a large overlap. A similar response may be originated by molecular dipoles in the vicinity of the rod, which suggests that dielectrically confined NRs are suited structures for sensing applications.

In conclusion, we have shown that the dielectric confinement of colloidal NRs leads to non-negligible configuration mixing effects, which are not captured by the usual singleparticle or perturbational descriptions of Coulomb interac- tions. The configuration mixing induces important changes in the few-body wave function, which have visible consequences on the optical and transport properties of NRs, including improved luminescence and sensitivity. Our results indicate that the optical properties of NRs may be engineered by controlling the regime of Coulomb interaction. Even though the calculations were carried out for a 12-nm-long CdSe NR, the findings apply also to different materials and correlations will be even more relevant for longer NRs.

\section{ACKNOWLEDGMENTS}

We wish to thank G. Goldoni for helpful comments. Support from MCINN project under Project No. CTQ200803344, UJI-Bancaixa project under Project No. P1-1B200603, the Ramon y Cajal program (J.I.C.), a Generalitat Valenciana FPI grant (M.R.), and Cineca Calcolo Parallelo is acknowledged. *josep.planelles@qfa.uji.es

${ }^{1}$ X. Peng, L. Manna, W. Yang, J. Wickham, E. Scher, A. Kadavanich, and A. P. Alivisatos, Nature (London) 404, 59 (2000).

${ }^{2}$ S. Kan, T. Mokari, E. Rothenberg, and U. Banin, Nature Mater. 2, 155 (2003).

${ }^{3}$ U. Woggon, J. Appl. Phys. 101, 081727 (2007), and references therein.

${ }^{4}$ H. Htoon, J. A. Hollingsworth, R. Dickerson, and V. I. Klimov, Phys. Rev. Lett. 91, 227401 (2003).

${ }^{5}$ M. A. El-Sayed, Acc. Chem. Res. 37, 326 (2004).

${ }^{6}$ D. Steiner, D. Katz, O. Millo, A. Aharoni, S. Kan, T. Mokari, and U. Banin, Nano Lett. 4, 1073 (2004).

${ }^{7}$ D. Katz, T. Wizansky, O. Millo, E. Rothenberg, T. Mokari, and U. Banin, Phys. Rev. Lett. 89, 086801 (2002).

${ }^{8}$ J. Hu, L. W. Wang, L. S. Li, W. Yang, and A. P. Alivisatos, J. Phys. Chem. B 106, 2447 (2002).

${ }^{9}$ J. Li and L. W. Wang, Nano Lett. 3, 101 (2003).

${ }^{10}$ J. Li and L. W. Wang, Nano Lett. 3, 1357 (2003).

${ }^{11}$ L. E. Brus, J. Chem. Phys. 80, 4403 (1984).

${ }^{12}$ V. A. Fonoberov, E. P. Pokatilov, and A. A. Balandin, Phys. Rev. B 66, 085310 (2002).

${ }^{13}$ N. A. Hill and K. B. Whaley, Phys. Rev. Lett. 75, 1130 (1995); A. Franceschetti and A. Zunger, ibid. 78, 915 (1997).

${ }^{14}$ M. Lannoo, C. Delerue, and G. Allan, Phys. Rev. Lett. 74, 3415 (1995).

${ }^{15}$ P. G. Bolcatto and C. R. Proetto, Phys. Rev. B 59, 12487 (1999).

${ }^{16}$ A. Shabaev and Al. L. Efros, Nano Lett. 4, 1821 (2004).

${ }^{17}$ L. V. Keldysh, Phys. Status Solidi A 164, 3 (1997).

${ }^{18}$ G. Goldoni, F. Rossi, and E. Molinari, Phys. Rev. Lett. 80, 4995 (1998).

${ }^{19}$ E. A. Muljarov, E. A. Zhukov, V. S. Dneprovskii, and Y. Masumoto, Phys. Rev. B 62, 7420 (2000).
${ }^{20}$ In Eq. (1), we have considered that the mixing of heavy-hole and light-hole subbands is weak. For the low-lying states of the structures, we study that this can be shown to be true.

${ }^{21}$ P. C. Sercel and K. J. Vahala, Phys. Rev. B 44, 5681 (1991).

${ }^{22}$ D. Boda, D. Gillespie, W. Nonner, D. Henderson, and B. Eisenberg, Phys. Rev. E 69, 046702 (2004).

${ }^{23}$ For the NR, the CI basis is built from the 28 (12) lowest singlehole (electron) spin orbitals with $m_{i}=0(i=e, h)$, the eight (four) lowest with $m_{i}= \pm 1$, and the four lowest with $m_{h}= \pm 2$. For the $\mathrm{NC}$, we use the eight (six) lowest spin orbitals with $m_{i}=0$, the six (four) lowest with $m_{e}= \pm 1$, the four (two) lowest with $m_{e}$ $= \pm 2$, and the two lowest with $m_{h}= \pm 3$.

${ }^{24}$ Electron-hole exchange and self-energy interactions are neglected as they do not influence the trends we report here. In particular, self-energy corrections consist roughly in a shift of the corresponding single-particle energy spectra, yielding minor effects on the Coulomb interactions. This is due to the nearly flat profile of the self-polarization potential in the medium with larger dielectric constant [see, e.g., L. Brus, J. Chem. Phys. 79, 5566 (1983)], which here is the NR.

${ }^{25}$ U. E. H. Laheld and G. T. Einevoll, Phys. Rev. B 55, 5184 (1997).

${ }^{26}$ J. S. Meyer and K. A. Matveev, J. Phys.: Condens. Matter 21, 023203 (2009).

${ }^{27}$ L. Jdira, P. Liljeroth, E. Stoffels, D. Vanmaekelbergh, and S. Speller, Phys. Rev. B 73, 115305 (2006).

${ }^{28}$ It is worth noting that our estimated enhancement of $V_{e h}$ for the NR $\left(265 \mathrm{meV}\right.$ at $\left.\varepsilon^{\text {out }}=1.75\right)$ is in the range of experimental measurements ( 240-300 meV) (Ref. 7).

${ }^{29}$ S. Corni, M. Brasken, M. Lindberg, J. Olsen, and D. Sundholm, Phys. Rev. B 67, 045313 (2003).

${ }^{30}$ L. Jacak, P. Hawrylak, and A. Wójs, Quantum Dots (SpringerVerlag, Berlin, 1998). 